\title{
PAPR Reduction for MIMO-OFDM Systems in Wireless Communications using PTS with DWT-DCT Scheme
}

\author{
Pranshu Shrivastava \\ M.E. Scholar \\ Department of Electronics \& Communication \\ University Institute of Technology, Bhopal, India
}

\author{
Anubhuti Khare, $\mathrm{PhD}$ \\ Professor \\ Department of Electronics \& Communication \\ University Institute of Technology, Bhopal, India
}

\begin{abstract}
Multiple Input Multiple Output (MIMO) in combination with Orthogonal Frequency Division Multiplexing (OFDM) is of great interest for researchers and research laboratories all over the world. OFDM is widely used in contemporary communication systems for its good robustness in multipath environment, and its high spectral efficiency. The capacity of wireless system can be increased dramatically by employing Multiple Input Multiple Output, (MIMO) antennas. The combination of MIMO and OFDM system is found to be very beneficial. A major drawback of OFDM-MIMO System is its high Peak to Average Power Ratio (PAPR) Reduction. The peak power of a signal is a critical design factor for band limited communication systems, and it is necessary to reduce it as much as possible. Many PAPR reduction techniques have been used to reduce PAPR. Partial transmit sequence (PTS) is one of the most well-known peak-to-average power ratio (PAPR) reduction techniques proposed for MIMO-OFDM systems. However the computational complexity of traditional PTS method is tremendous. In this paper a new partial transmit sequence (PTS) technique, based on PTS with discrete wavelet transform (DWT) and discrete cosine transform (DCT) technique, for two antennas MIMO-OFDM system, is proposed which can achieve better PAPR performance at much less bit error rate (BER). Simulation results show that the proposed approach can reduce BER and achieve a better PAPR reduction compared to previous PTS technique.
\end{abstract}

\section{Keywords}

PTS, DWT, DCT, MIMO-OFDM, PAPR, BER

\section{INTRODUCTION}

A combination of multiple input multiple output (MIMO) and orthogonal frequency division multiplexing (OFDM) (MIMOOFDM) is an emerging technology for high speed data multicarriers transmission in future wireless communication network systems such as digital audio broadcasting (DAB), digital video broadcasting (DVB), medical body area networks (MBANs) applications, the fourth and the fifth generation $(4 \mathrm{G}, 5 \mathrm{G})$ of mobile network. In MIMO-OFDM system, the output is the superposition of multiple sub-carriers. Whenever, the phases and frequencies of these carriers match coherently, instantaneous power outputs may increase greatly and become higher than the mean power of the high power amplifier (HPA) resulting in large PAPR [1]. Lot of research work has been done for solving the problem of PAPR that concerns all kind of multicarrier signals. So, many techniques have been proposed such as clipping [2], tone reservation [3], nonlinear transformations [4], coding [5], selecting mapping (SLM) [6] and partial transmit sequence (PTS). Modified approaches of PTS are proposed in that produce better results; however, the computational complexity is still remaining unsolved totally. In this paper an approach is proposed to reduce the PAPR in STBC MIMO-
OFDM systems with less computational complexity. So, the mean idea is based on PTS with discrete wavelet transform (DWT) and discrete cosine transform (DCT). The rest of the paper is organized as follows: in section II, MIMOOFDM system is explained. Section III describes the PTS algorithm. Section IV shows the proposed algorithm, simulation results and the paper are concluded in section V and section VI.

\section{MIMO-OFDM SYSTEM}

MIMO in combination with OFDM is widely used nowadays due its best performance in terms of capacity of channels, high data rate and good outcome in frequency selective fading channels [7]. Notwithstanding this it additionally enhances unwavering quality of connection. This is accomplished as the OFDM can change recurrence particular MIMO channel to recurrence level MIMO channels [8]. So it is generally utilized as a part of future broadband remote framework/interchanges. Cyclic prefix is the copy of last part of OFDM symbol which is appended to the OFDM symbol that is to be transmitted. It is basically $0.25 \%$ of the OFDM symbol. It can say that one fourth of the OFDM symbol is taken as $\mathrm{CP}$ (cyclic prefix) and appended to each OFDM symbol. IFFT is used at the transmitter and FFT is used at the receiver which substitutes the modulators and demodulators. Doing so eliminates the use of banks of oscillators and coherent demodulators. Moreover the complex data cannot be transmitted as it is; therefore it is first converted to analog form which is accomplished by IFFT. It basically converts the signal from frequency domain to time domain. Prior to IFFT operation symbol mapping is performed which is nothing but the modulation block. Any of the widely used modulation techniques can be applied like BPSK, QPSK, QAM, PSK etc. Further there are higher order modulations are also available which provide more capacity at little expense of BER performance degradation [9]. After IFFT piece pilot addition is done and afterward CP (cyclic prefix) is included. Figure 1 underneath demonstrates the piece outline constituting MIMO and OFDM. Any receiving wire arrangement for the MIMO can be utilized by the framework prerequisite. Higher the configuration more will be the capacity and more will be the computational complexity of the transceiver design. It is seen that in the case of estimating channel the computational complexity is increased. Mapper defines the modulation to be used. Symbol encoder takes the shape of the STBC (Space Time Block Code) if spatial diversity is to be used and it takes the shape of the de-multiplexer/multiplexer if spatial multiplexing is to be used. 


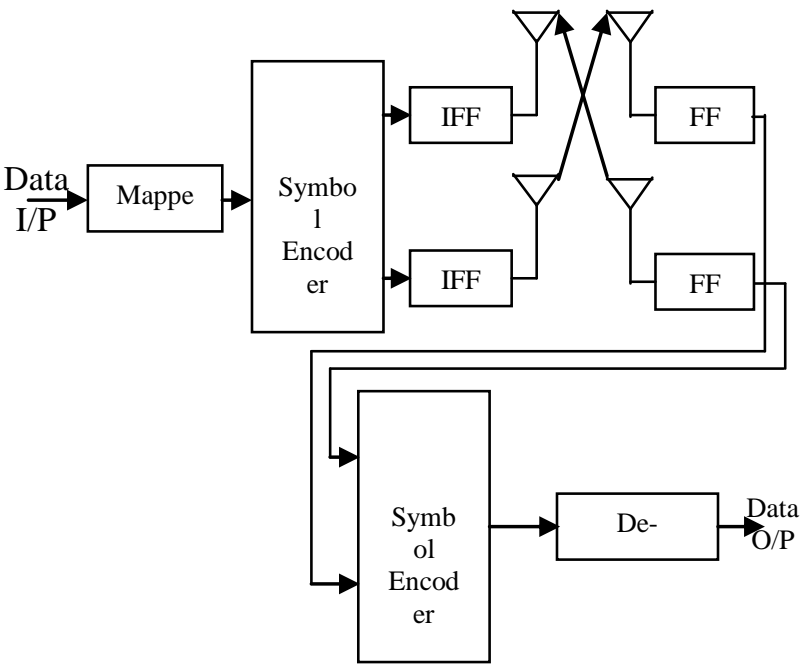

Figure 1: MIMO-OFDM system model

The received signal at $\mathrm{j}^{\text {th }}$ antenna can be expressed as

$$
\mathrm{R}_{\mathrm{j}}[\mathrm{n} \cdot \mathrm{k}]=\sum \mathrm{H}_{\mathrm{ij}}[\mathrm{n}, \mathrm{k}] \mathrm{X}_{\mathrm{i}}[\mathrm{n}, \mathrm{k}]+\mathrm{W}[\mathrm{n}, \mathrm{k}]
$$

Where $\mathrm{H}$ is the channel matrix, $\mathrm{X}$ is the input signal and $\mathrm{W}$ is noise with zero mean and variance. Also $b_{i}[n, k]$ represents the data block $i^{\text {th }}$ transmit antenna, $\mathrm{n}^{\text {th }}$ time slot and $\mathrm{k}^{\text {th }}$ sub channel index of OFDM. Here $i$ and $j$ denoted the transmitting antennas index and receiving antenna index respectively.

The MIMO-OFDM system model [10] with NR receives antennas and NT transmits antennas can be given as:

$$
\left[\begin{array}{c}
Z_{1} \\
Z_{2} \\
\vdots \\
Z_{N}
\end{array}\right]=\left[\begin{array}{ccccc}
H_{1,1} & H_{1,2} & \ldots & H_{1, N T} \\
H_{2,1} & H_{2,2} & \ldots & H_{2, N T} \\
\vdots & \vdots & \ddots & \vdots \\
H_{N R, 1} H_{N R, 2} & \ldots & H_{N R, N T}
\end{array}\right]\left[\begin{array}{c}
A_{1} \\
A_{2} \\
\vdots \\
A_{N T}
\end{array}\right]+\left[\begin{array}{c}
M_{1} \\
M_{2} \\
\vdots \\
M_{N T}
\end{array}\right]
$$

(2)

Where, $\mathrm{Z}$ represents $\mathrm{O} / \mathrm{P}$ data vector, $\mathrm{H}$ denotes Channel matrix, A denotes I/P data vector and $\mathrm{M}$ represents Noise vector. The wireless channel used is AWGN channel. After receiving the signal the $\mathrm{CP}$ is removed then the pilots are also removed from main signal received. After this the signal that is in time domain can be again converted to frequency domain by taking FFT of the received signal [10].

The sequence on each of the OFDM block is then provided to channel estimation block where the received pilots altered by channel are compared with the original sent pilots. Channel estimation block consists of the algorithms that are applied to estimate the channel.

\section{PTS SCHEMES}

In the SISO-PTS scheme, the original data sequence in the frequency domain is partitioned into $\mathrm{M}$ disjoint, equal length sub blocks $\mathrm{Xv}(\mathrm{v}=1,2 \ldots \mathrm{M})$ as follows [11].

$$
X=\sum_{v=1}^{M} X_{v}
$$

By multiplying some weighting coefficients to all the subcarriers in every sub-block, it can get the new frequency sequence.
$X^{\prime}=\sum_{v=1}^{M} b_{v} X_{v}$

Finally, at each transmitting antenna, there are (V-1) sub blocks to be optimized, and the candidate sequence with the lowest PAPR is individually selected for transmitting. Assume that there are $\mathrm{W}$ allowed phase weighting factors. To achieve the optimal weighting factors for each transmitting antenna, combinations should be checked in order to obtain the minimum PAPR [12].

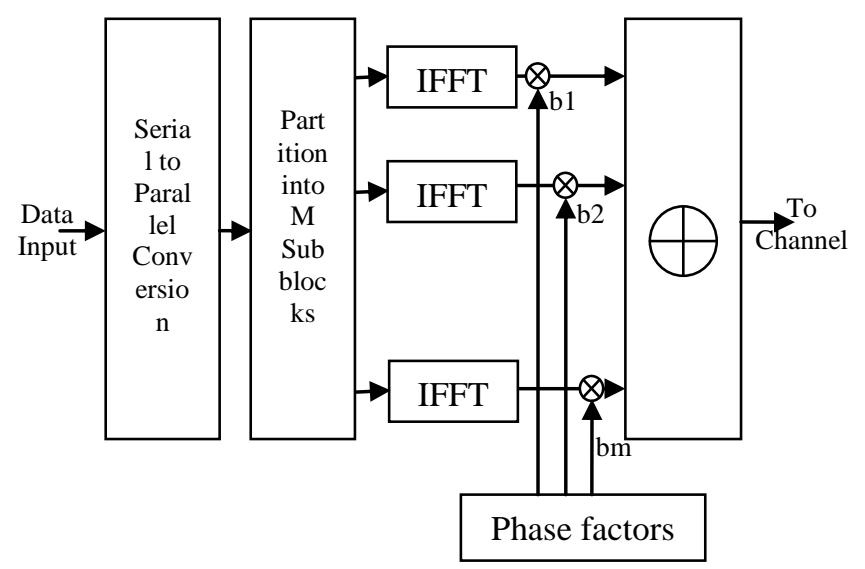

Figure 2: Block Diagram of PTS Scheme

In, the idea of alternate optimization is introduced, and it can be also applied to PTS in multiple antennas OFDM systems, denoted as alternate PTS. Different from ordinary PTS, phase weighting factors are needed only for half of the sub blocks in A-PTS. That is to say, starting from the first sub block, every alternate sub block is kept unchanged and phase weighting factors are optimized only for the rest of the sub blocks, which leads to the reduction of computational complexity. In this way, the computational complexity is greatly reduced at the expense of PAPR performance degradation [11]. Employed spatial sub block circular permutation for A-PTS scheme to increase the number of candidate sequences which improves the PAPR performance further.

Next, the conversion of the optimum weighting coefficient is discussed. In order to maintain the conjugate and symmetric relations between the two antennas after scrambling sequence methods, it should convert the optimum weighting coefficient a(opt) at antenna 1 into that of antenna 2 denoted as b(opt) by the inverse conjugate and symmetric transformation. For example, when the optimum weighting coefficient a (opt) is $[1$, $1, \mathrm{j},-\mathrm{j}]$, the optimum weighting coefficient for antennas 2 is $\mathrm{b}$ (opt) $=[1,1,-\mathrm{j}, \mathrm{j}]$. The PTS scheme can be also applied to the MIMO-OFDM system with more transmits antennas.

Based on advance PTS, an approach to solve the contradiction between the PAPR performance and computational complexity in STBC MIMO-OFDM system is proposed. Let us consider a STBC MIMO-OFDM system that employs Alamouti scheme. The coding matrix is:

$G=\left(\begin{array}{cc}x_{1} & -x_{2}^{*} \\ x_{2} & x_{1}^{*}\end{array}\right)$

Simulation experiments are conducted to evaluate the transmit spectrum, bit error rate (BER), peak average to peak ratio (PAPR) reduction performance of the MIMO-OFDM scheme 
using PTS technique. In addition, it is assumed that the data are QPSK, 16-QAM modulated and are transmitted using 256 FFT.

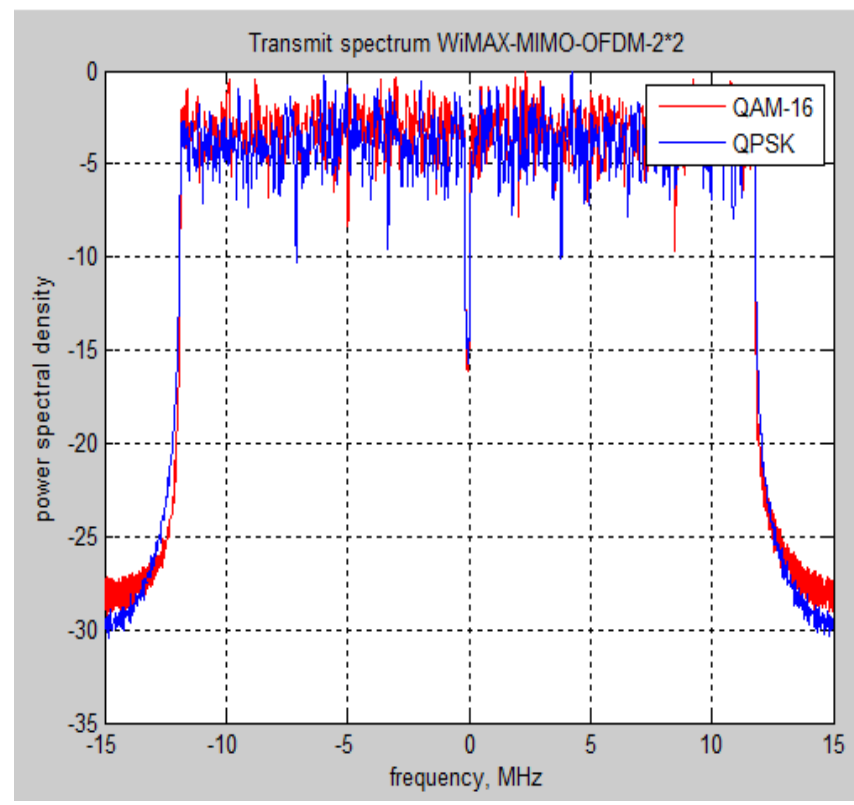

Figure 3: Power Spectral Density of MIMO-OFDM $2 \times 2$ System

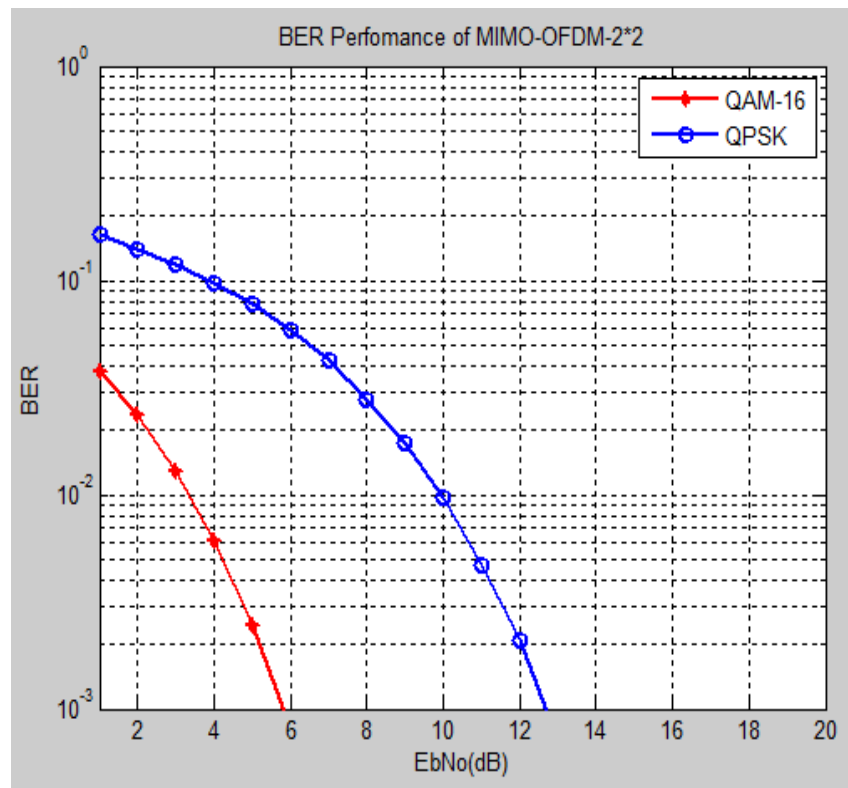

Figure 4: BER Performance of MIMO-OFDM $2 \times 2$ System

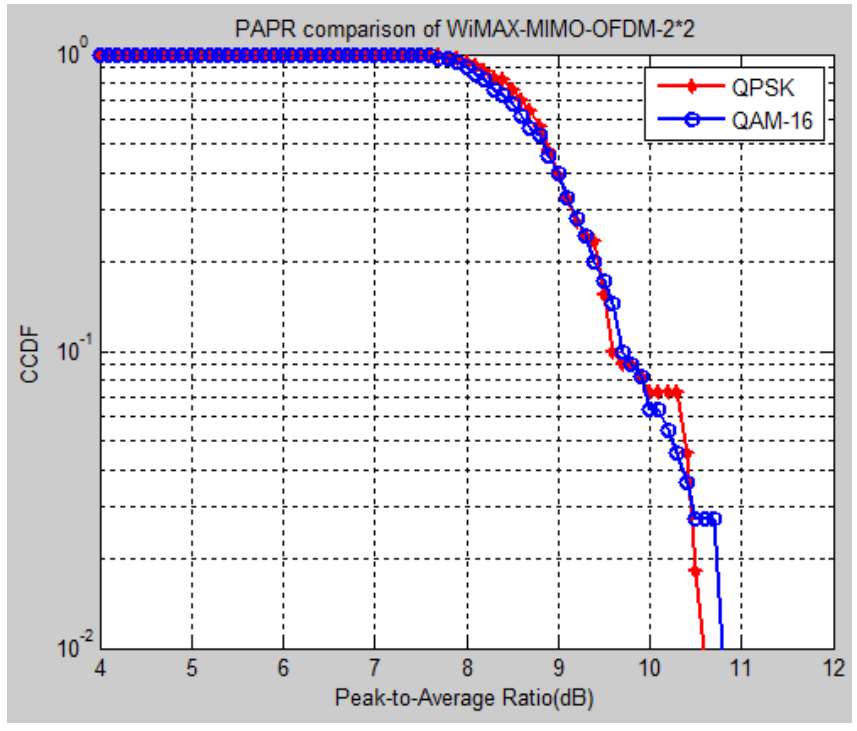

Figure 5: PAPR Performance of MIMO-OFDM $2 \times 2$ System

\section{PROPOSED METHODOLOGY}

The proposed a wavelet based MIMO-OFDM system for the reduction of PAPR, which effectively reduces the PAPR on rational selection of phase values is presented. First the original input signal is modulated with BPSK, QPSK, QAM-16 and PTS technique had been applied, where the phase values are generated using optimized algorithm. This helps to minimize the PAPR of the input signal. Then discrete wavelet transform is applied and has been followed by DCT which is applied transmitted through AWGN channel. At the receiver, the inversion of transmitter will be done.

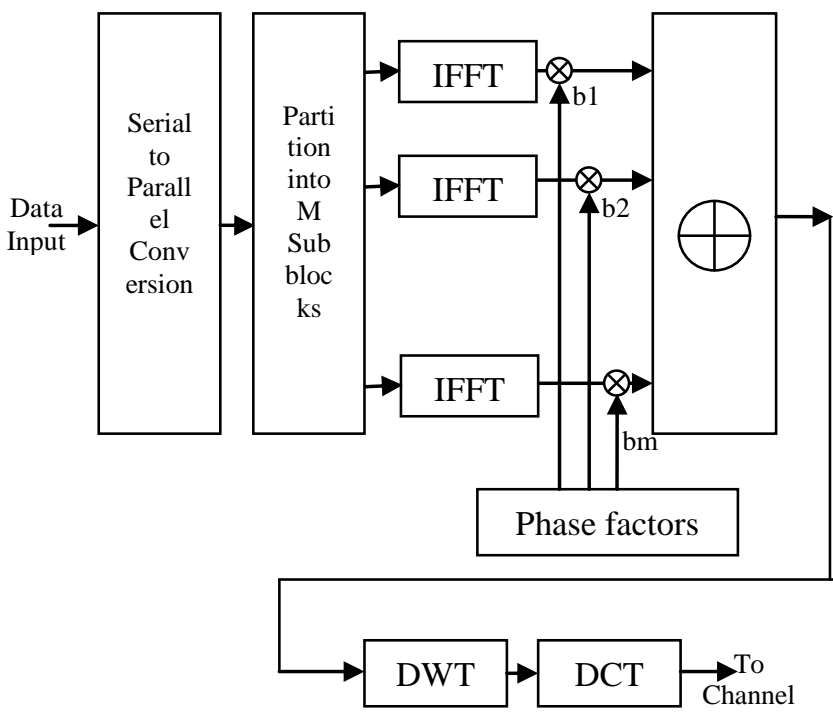

Figure 6: Flow Char of Proposed Methodology

Figure 6 shows the enhanced transmitter block diagram of the presented work. In this research work conventional OFDM is followed by the WPT and DCT for PAPR reduction and vice versa is also simulated. Both transmitter and receiver are simulated in order to calculate the BER.

\section{SIMULATION RESULT}

The CCDF is generally used to evaluate the performance of PAPR reduction on MIMO-OFDM system (IEEE 802.16e) signals for a statistical pair of view. The CCDF is defined as the 
probability that the PAPR as in equation and $\mathrm{PAPR}_{0}$ as shown in the following:

$$
P A P R\{Y\}=\arg \underbrace{\max }_{k=1,2,3, \ldots \ldots N_{T}}\left(P A P R\left\{Y_{k}\right\}\right)
$$

Where $Y_{k}, k=1.2 .3 \ldots \ldots . . N_{T}$ represents the time-domain transmitted signal of the $\mathrm{k}$-th antenna

$$
C C D F\left(P A P R_{0}\right)=\operatorname{Pr}\left(P A P R\{Y\}>\left\{P A P R_{0}\right\}\right)
$$

Figure 7 shows the graphical illustration of the performance of MIMO-OFDM $2 \times 2$ System using PTS with DWT-DCT technique discussed in this research work in term of bit error rate (BER). From the above graphical representation it can be inferred that the proposed algorithm gives the best performance for QAM-16 modulation technique.

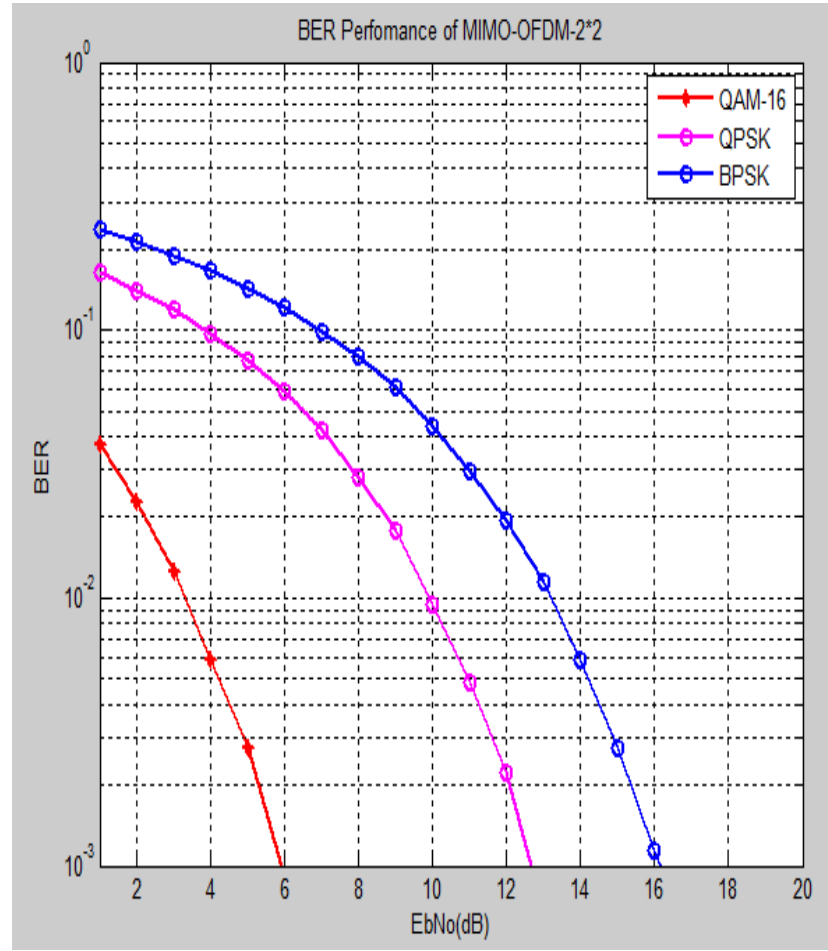

Figure 7: BER Performance of MIMO-OFDM $2 \times 2$ System

Figure 8 shows the graphical illustration of the performance of MIMO-OFDM $2 \times 2$ System discussed in this research work in term of peak signal to noise ratio (PAPR). From the above graphical representation it can be inferred that the proposed PTS with DWT and DCT technique based MIMO-OFDM algorithm gives the best performance for QAM-16 modulation technique.

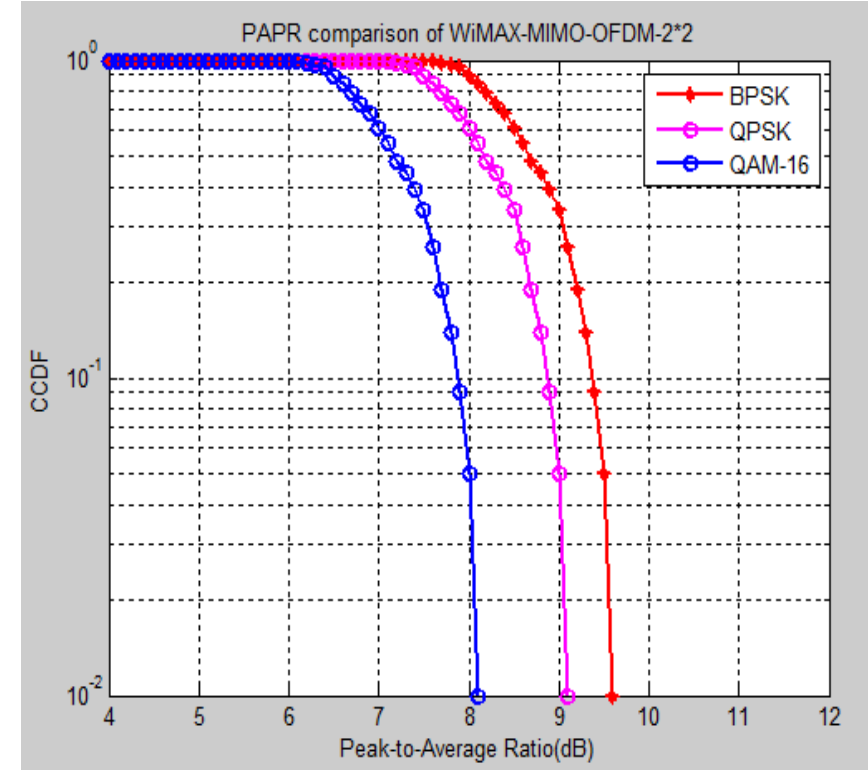

Figure 8: PAPR Performance of MIMO-OFDM $2 \times 2$ System

Table 1: Comparison of PAPR (dB) values for Hybrid, PTS and original PAPR signal for FFT SIZE 256

\begin{tabular}{|l|l|l|l|}
\hline $\begin{array}{l}\text { Original } \\
\text { PAPR }\end{array}$ & $\begin{array}{l}\text { PTS } \\
\text { Technique }\end{array}$ & $\begin{array}{l}\text { Hybrid PTS } \\
\text { with } \\
\text { Clipping } \\
\text { technique }\end{array}$ & $\begin{array}{l}\text { PTS with } \\
\text { DWT-DCT } \\
\text { technique }\end{array}$ \\
\hline $10.3 \mathrm{~dB}$ & $8.7 \mathrm{~dB}$ & $8.5 \mathrm{~dB}$ & $8.0 \mathrm{Db}$ \\
\hline
\end{tabular}

Table II: Comparison of BER (dB) Values for Hybrid, PTS

\begin{tabular}{|c|c|c|c|}
\hline \multicolumn{4}{|l|}{ BER (dB) } \\
\hline $\begin{array}{l}\text { Energy per } \\
\text { bit (EbNo) }\end{array}$ & BPSK & QPSK & QAM-16 \\
\hline $10^{-3}$ & 16.1 & 12.2 & 6 \\
\hline $10^{-2}$ & 13.2 & 10 & 3.5 \\
\hline $10^{-1}$ & 7 & 4 & 0 \\
\hline $10^{-0}$ & 0 & 0 & 0 \\
\hline
\end{tabular}
and Original Signal

\section{CONCLUSION}

MIMO-OFDM is a very agreeing method for the new wireless digital communication system. Along with the simplicity of equalization in Orthogonal Frequency Division Multiplexing (OFDM) modulation, it combines the capacity and diversity gain of MIMO systems for better performance. However, like conventional OFDM, MIMO-OFDM has a major challenge called high PAPR. Hence, it requires high dynamic range power amplifier, which makes more cost of system and decreases the efficiency of power. In this paper, a method is proposed for minimization of PAPR in MIMO-OFDM systems using PTS method. The PTS is concatenated with DCT and DWT signal processing algorithm to improve the efficiency and reduction of peak power of the MIMO-OFDM system. Because of autocorrelation of DCT the average power will be reduced. Using DWT, the data is divided into high and low coefficient such that the cyclic prefix can be avoided. By using PTS 
method, the optimum phase's factors are selected to reduce the average power. Hence it is achieved that MIMO- OFDM signals with less PAPR.

\section{REFERENCES}

[1] Ashna Kakkar, Sai Nitesh Garsha,Ojasvi Jain and Kritika, "Improvisation in BER and PAPR by using hybrid reduction techniques in MIMO-OFDM employing channel estimation techniques", 2017 IEEE 7th International Advance Computing Conference.

[1] S. H. Han, J.H. Lee, " An Overview of Peak Average Power ratio Reduction techniques for Multicarrier Transmission", IEEE wireless Communications, Vol. 25, pp. 56-65, March, 2005H.

[2] H. Yang, "A Road to Future Broadband Wireless Access: MIMOOFDM based Air Interface, "IEEE Communications Magazine, Vol. 43, No. 1, pp.53-60, 2005.

[3] Tarokh, N. Seshadri, and A. R. Caderbank, " SPACE-TIME Codes for High Data Rate Wireless Communication: Performance Criteria and Code Construction," IEEE Trans. Inform. Theory, pp. 744-764, Mar. 1988.

[4] H. Ochiai and H. Imai. Performance of the deliberate clipping with adaptive symbol selection for strictly bandlimited OFDM systems. IEEE Journals on Selected Areas in Communications, 18(11):2270-2277, November 2000.

[5] Jose Tellado. Peak to Average Power Ratio Reduction for Multicarrier Modulation. $\mathrm{PhD}$ thesis, University of Stanford, Stanford, 1999.
[6] Tao Jiang and Guangxi Zhu. Nonlinear companding transform for reducing peak-to average power ratio of OFDM signals. Broadcasting, IEEE Transactions on, 50(3):342 - 346, sept. 2004.

[7] S. B. Slimane. Reducing the peak-to-average poser ratio of OFDM signal through precoding. IEEE Trans Vehicular Technology, 56(2) 686-695, mar 2007.

[8] R.W. Bauml, R.F.H. Fisher and J.B. Huber, Reducing the peak-to average power ratio of multicarrier modulation by selected mapping[J] IEEE Electronic Letter, 1996, 32(22): 2056-2057.

[9] S.H. Muller and J.B. Huber, OFDM with reduced peak-toaverage power ratio by optimum combination of partial transmit sequences [J]. IEEE Electronic Letter, 1997, 33(5): 368-369.

[10] L. Yang, K. K. Soo, S. li, and Y. M. SU, “PAPR Reduction Using Low Complexity PTS to Construct of OFDM Signals Without Side Information", IEEE Transactions on Broadcasting, Vol. 57, No. 2, June 2011.

[11] D.W Lim, S.J.Heo and J.S.No, A new PTS OFDM scheme with low complexity for PAPR reduction [J]. IEEE Transactions on Broadcasting, 2006, 52(1): 77-82.

[12] P. Mukunthan and, P Dananjayan," PAPR Reduction based on a Modified PTS with Interleaving and Pulse Shaping method for STBC MIMO-OFDM System", IEEE ICCCNT'12, 26th_28th July 2012, Coimbatore, India. 